\title{
Auflistung häufiger Auslagen der Praxis
}

Nach § 10 GOÄ können die Kosten, die aus der individuellen Behandlung eines Patienten zusätzlich entstehen z. B. für Arzneimittel, Verbandmittel und sonstigen Materialien, die der Patient zur weiteren Verwendung behält oder die mit einer einmaligen Anwendung verbraucht sind, als Auslagen berechnet werden.

Die Kosten für berechnungsfähig verbrauchte Materialien gem. § 10 GOÄ sind am Schluss der Rechnung detailliert anzugeben. Wird der Betrag von $€ 25,56$ überschritten, so ist auch ein Rechnungsbeleg beizufügen.

Rabatte, die ein Hersteller oder auch ein Apotheker wegen z. B. des Bezuges größerer Mengen gewährt, müssen an den Zahlungspflichtigen weitergegeben werden.

Die Berechnung von Pauschalen ist nicht zulässig.

Die beigefügten Belege können sich auch auf größere Liefermengen (als nur für einen Patienten) und dadurch größere Beträge belaufen Aus dem Beleg muß die bezogene Menge hervorgehen und aus der Honorarrechnung des Arztes muß die im konkreten Behandlungsfall verbrauchte Teilmenge deutlich hervorgehen.

Nicht berechnet werden können die Kosten für

1. Kleinmaterialien wie Zellstoff, Mulltupfer, Schnellverbandmaterial, Verbandsspray, Gewebeklebstoff auf Histoacrylbasis, Mullkompressen, Holzspatel, Holzstäbchen, Wattestäbchen, Gummifingerlinge,

2. Reagenzien und Narkosemittel zur Oberflächenanästhesie,

3. Desinfektions- und Reinigungsmittel,

4. Augen-, Ohren-, Nasentropfen, Puder, Salben und geringwertige Arzneimittel zur sofortigen Anwendung sowie für

5. folgende Einmalartikel: Einmal-Spritzen, -Kanülen, -Handschuhe, -Harnblasenkatheter, -Skalpelle, -Proktoskope, -Darmrohre, -Spekula.

\section{Rezeptieren von Material}

Meistens rezeptieren Ärzte im Rahmen von IGeL-Leistungen auf einem Privatrezept die erforderlichen Medikamente (Arzneimittel-Ampullen, Infusionslösungen u. a.) und das ggf. notwendige Verbandsmaterial sowie z. B. Braunülen, Infusionsbestecke etc. Der Patient besorgt dies in der Apotheke und zahlt es auch. Dies stellt für den Arzt - der diese Auslagen in der Liquidation nicht aufführen muss - eine Vereinfachung dar.

\section{Eine Liste für den Praxisalltag}

Für häufig verwendete z. B. Arzneimittel, Verbandmittel und sonstigen Materialien ist eine Aufstellung ihrer (Einzel-)Preise in Zusammenarbeit z. B. mit einem Apotheker sinnvoll und erleichtert Nachbestellungen und hilft bei Rechnungstellungen keine Auslagen zu vergessen. In die folgende Liste können Sie Ihre praxisindividuellen Auslagen eintragen: 